\title{
The design of the digital power supply for the airbag static deployment test
}

\author{
LV Li Ya ${ }^{1, a}$, MIN Yong Jun ${ }^{1, b}$ \\ ${ }^{1}$ College of Automobile and Traffic Engineering, Nanjing Forestry University, Nanjing, China \\ avliya@163.com, byjmin@njfu.com.cn
}

Keywords: Automobile Electronics; Air bag; Embedded System; Numerical control Power

\begin{abstract}
The author of this paper in the process of detonation possible problems: not accident when explosive accident, when not explosion point or not, the airbag static expansion needed power supply parameters, can be designed for air bag deployment time, adjustable current size parameters of embedded power supply system. Experimental platform through touch human-machine interface set the current and voltage values of pulse delay time, the retention time of the pulse, simulated airbags in automobile traffic accident to detonate the signal, the realization of static detonate airbag, and triggers the flash light and high-speed cameras record the airbag detonation process. By actual airbag deployment test, this system has reached the experiment purpose, for the airbag experiment and examination provides the intelligent, generalized solution.
\end{abstract}

\section{Introduction}

Airbag as a kind of electromechanical product, with the increase in car ownership, more and more quality problems expose in all kinds of traffic accidents and complaints and reports about the quality of the airbags are increasing. Protection ability of the airbag is related to the work in the event of a collision, but also with the process and scope of the airbag deployment. Some passengers get hurt in a collision and it is from the impact of the airbag itself. Therefore, the detection of the state and process of the airbag deployment is beneficial to improve the safety performance of the airbag. This paper mainly focuses on determining the system scheme for static deployment test device of the airbag by understanding the test requirements of the automobile airbag static deployment test ${ }^{[1]}$. On this basis, to study the airbag power parameters required for static deployment, and design the embedded power system which can be adjusted by the time and the adjustable current parameters of the airbag ${ }^{[2]}$.

\section{The design scheme of airbag-igniting numerical control power supply}

Airbag-igniting numerical control power supply is mainly composed of MCU numerical control module, constant current flow module, power module, communication module, touch display module and so on. Among them, the power module turns AC $220 \mathrm{~V}$ voltage into system working required $5 \mathrm{~V}$ and $12 \mathrm{~V}$ through the regulator, and then supplies each system respectively. This system is designed in a very low temperature coefficient, low aging characteristics of the voltage reference circuit. It adopts high speed constant current module with negative feedback, by filtering the output voltage and current of the power supply. The output of the system is a constant current signal. The system output is program-controlled constant-current signal. In addition, the system uses the unique SYSTICK clock technology in ARMMCU, and realizes the accurate pulse burst time of the airbag. Meanwhile, the communication system uses MAX232 chip and make MCU and the touch module for real-time data exchange, and it will feedback the output value to the touch screen. The system also adds the temperature detection section, using LM35 temperature sensor to monitor the power supply operating temperature. If the temperature is too high, then open the fan for cooling and the temperature can control fan on/off for overheating protection, which can realize the long time reliability test of the airbag.The overall diagram of igniting numerical control power supply is shown in Figure 1. 


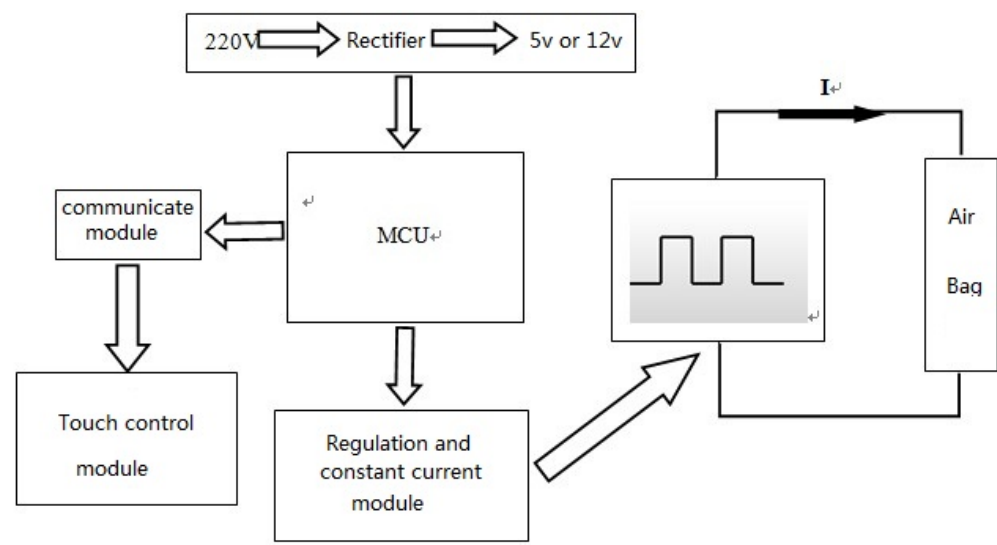

Figure1 Functional block diagram of airbag static igniting power supply

\section{The igniting numerical control power supply circuit design}

The igniting numerical control power supply uses 32 bit ARM processor STM32F103VET6, the chip is integrated with 12 bit ADC and DAC module, which greatly simplifies the peripheral circuit design of the trigger power, and the volume and power consumption of the system is very low; Due to the integrated ADC and DAC can realize the detection and display of the input and output signals conveniently inside of MCU through software algorithms and can achieve higher accuracy at the same time ${ }^{[3]}$.

\section{ADC and DAC circuit parameter design}

Figure 2 is output voltage feedback and acquisition circuit. Due to the real-time detection and control of the output signal, and the igniting output voltage range is $0-10 \mathrm{~V}$, which does not meet the needs of CPU AD range $(0-3.3 \mathrm{~V})$, so we use the forward amplifier for amplifying the output voltage in the design. For output voltage $\mathrm{V}_{\mathrm{O}}$, first of all, it inputs voltage of the operational amplifier after resistance voltage division,

$$
\operatorname{Vin}(\mathrm{MAX})=\mathrm{Vo} * \mathrm{R} 8 /(\mathrm{R} 2+\mathrm{R} 8)=9 * 10 / 53=1.7 \mathrm{~V}
$$

magnification times forward operational amplifier are as follows:

$$
\mathrm{A}=1+\mathrm{R} 22 / \mathrm{R} 24=1.23
$$

So positive amplification of the acquisition voltage $V_{A D-V}=1.7 \times 1.23=2.091 V$, it can meet the range of $\mathrm{AD}$.

Figure 3 is output current sampling circuit, according to the design requirements, current output range is $0 \sim 4 \mathrm{~A}$ and sampling resistance is $0.047 \Omega$, magnification times of amplifying circuit:

$$
\mathrm{A}=(\mathrm{R} 33+\mathrm{R} 34) / \mathrm{R} 35=122 \mathrm{~K} / 10 \mathrm{~K}=12.2
$$

So the sampling values of output signal are:

$$
\mathrm{V}_{\mathrm{AD}-\mathrm{A}}=4 * 0.047 * 12.2=2.29 \mathrm{~V}
$$

Can meet the conversion range of $\mathrm{AD}$. 


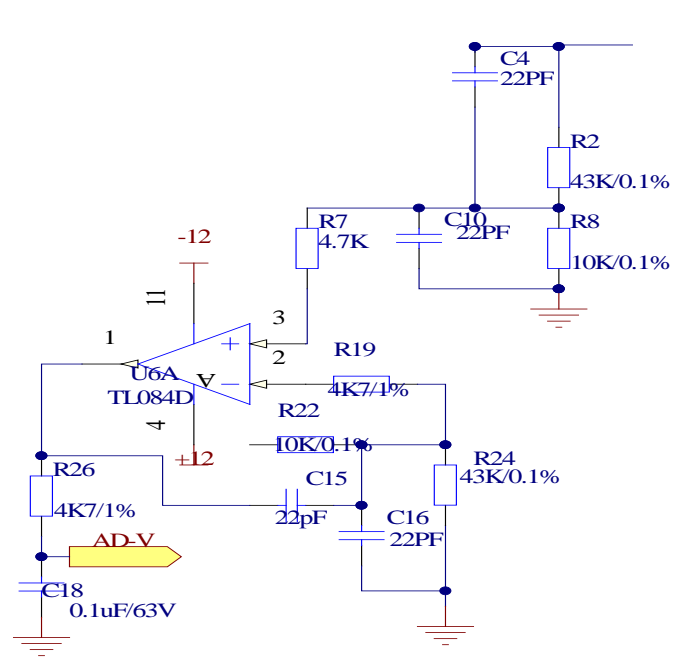

Figure 3 Current output sampling circuit

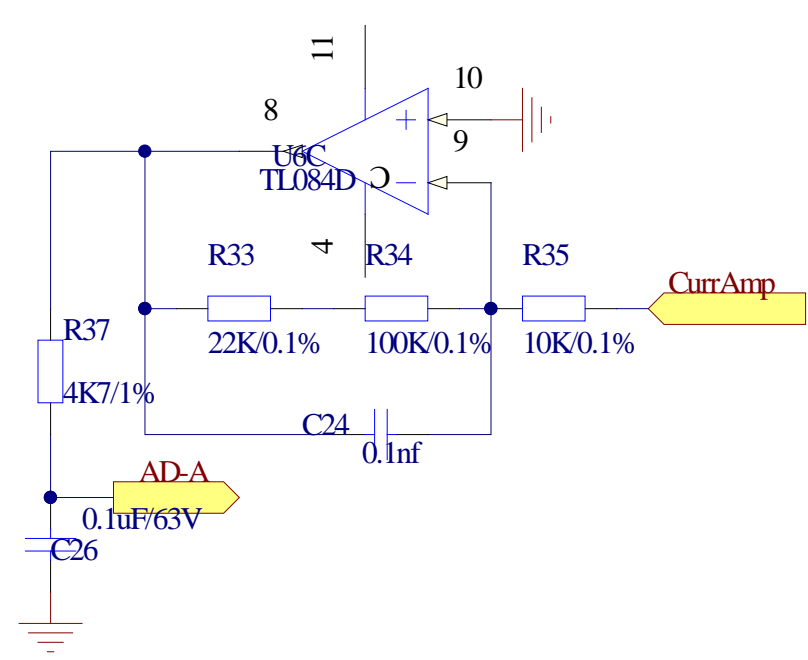

Figure 2 Voltage output sampling circuit

\section{Power amplification circuit parameter design}

Figure 4 is a power amplifier circuit and it achieves the control of the gate-source voltage of the field effect transistor through the CPU control power amplifier in order to realize the adjustment of the output signal. In the design, we use the positive and negative power supply to constitute the complementary symmetrical power amplifier circuit as showed above. Q4, Q3 are respectively NPN type tube and PNP tube, the base and emitter of the two tubes are respectively connected with the signal from the base input, output from emitter. And to add two resistances between the two tube emitter in order to form negative feedback, to reduce the distortion and improve the stability of the circuit, to prevent thermal breakdown, to suppress the no-load current with the temperature change [4].

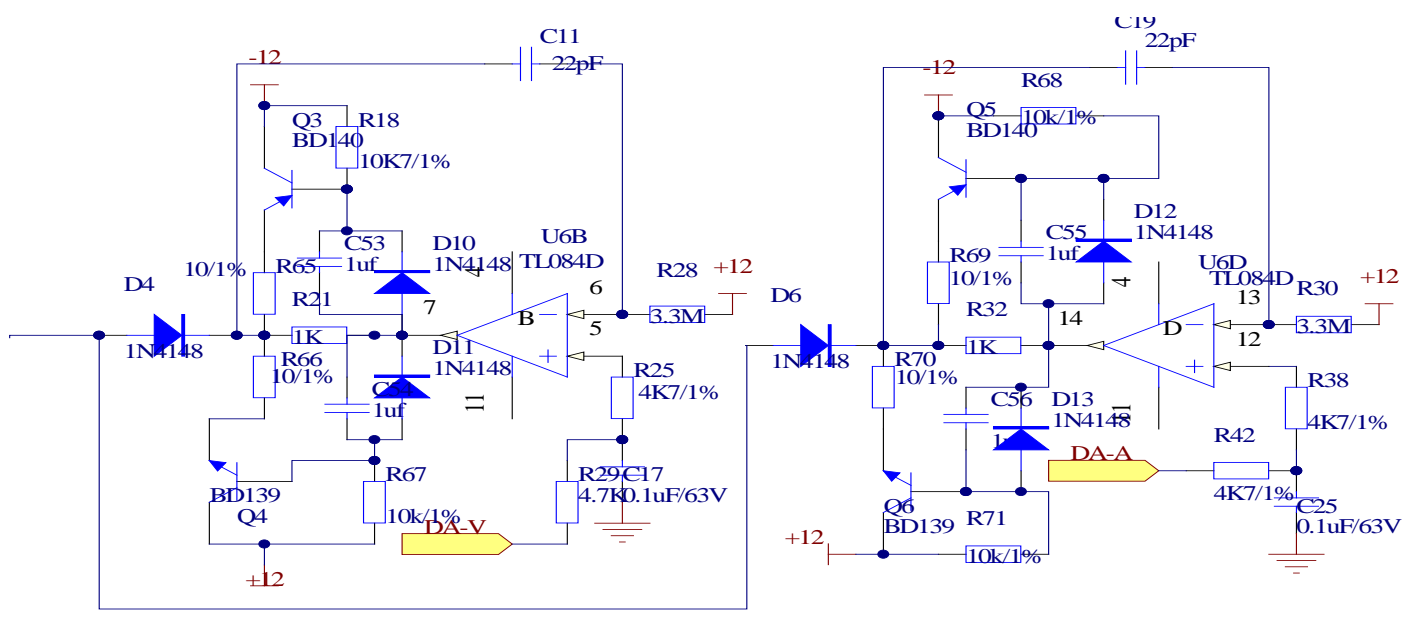

Figure 4 Voltage negative feedback and power amplifier circuit

At the same time, the project has a very high stability requirement on the output current. Therefore, it requires a high precision sampling resistor. We adopt the German ISA high precision resistor $(0.047 \Omega \pm 0.5 \%)$, it has a low temperature coefficient, low thermal voltage, low self inductance, long-term stability and high pulse can increase load capacity. Figure 5 is current sampling circuit. Considering the voltage loss of the wire between the trigger circuit and the airbag, it designs the remote voltage acquisition interface.

\section{Cooling and intelligent temperature control circuit}

Airbag-igniting requires large current output, power supplies in parallel of two MOSFET as the output tube, which increases its output capacity and reduces internal resistance of power. According to MOS tube characteristics, when the current increases, the pipe temperature rises, its resistance will increase as well. When the current in a pipe is too large, the temperature rises and theresistance increases. For parallel circuit, the electric resistance is larger and the electric current will be small. 
The output current of the circuit is stable. Therefore, if many pipes connected in parallel, there will be the effect of the automatic flow. In order to realize the reliability test of the safety airbag, the aluminum block is installed on the MOSFET. The fan is installed and it realizes the temperature control circuit. The high precision temperature sensor LM35 is used to avoid the possibility of the damage.

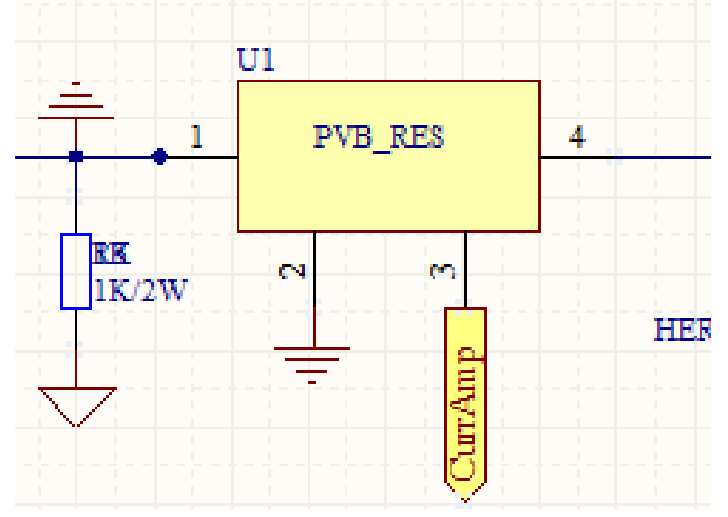

Figure 5 Current sampling circuit

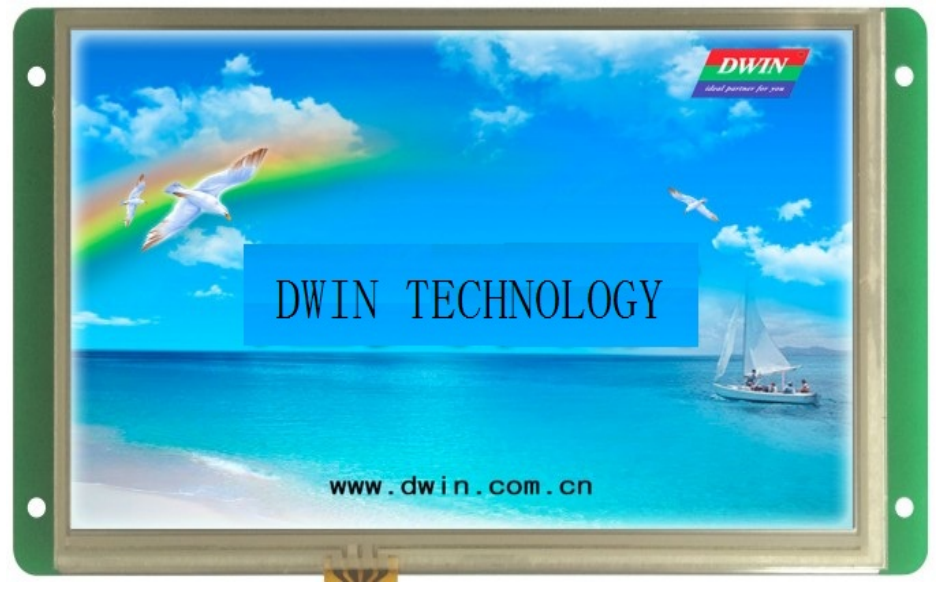

Figure 6 Dwin man-machine interface figure

\section{Output power switch circuit design}

The power supply in the design uses a high-speed, high current, photoelectric isolation PHOTO-MOSFET switch. It realized the high current no spark of high-speed switch, switch response time is less than $1 \mathrm{~ms}$. Meanwhile, it can realize the flexible switch strategy through the software design. This project adopts the AQZ192 PHOTO-MOSFET which produced by Panasonic Corp. And its maximum output current is $10 \mathrm{~A}$, which meets the requirements of the airbag-igniting current. At the same time, the power supply is also integrated with external flash, high speed camera trigger circuit.

\section{Airbag-igniting power supply software design}

The power system design uses the touch control interface and the design of serial communication protocol. It realizes the real-time input of all control parameters through the touch screen, and the system's internal parameters, output voltage current and other data can be displayed in real time on the man-machine interface.

\section{Man-machine interface software design}

Using the new touch screen man-machine interface as the control panel, it has the advantages of clear display, easy input, convenient data observation, convenient software upgrade, short development cycle and simple design. Touch human-machine interface model is: Beijing Dwin DMT80480S070_06W, its parameters are 7 "800 * 480 graphic dot matrix K600 + kernel 65K color DGUS screen and its shape is as shown in Figure 6.

General instrument display interface development needs professional software, such as $\mathrm{VC}++6.0$, etc. The cycle is long and the cost is high. And this project only needs to design the interface and the control design software of the man-machine interface. The output interface of the project uses LABVIEW software. LABVIEW has good GUI design function, which can quickly and beautifully design the appearance and function of the instrument. In this project, the display interface software and the principle diagram of the main control is shown in Figure 7 


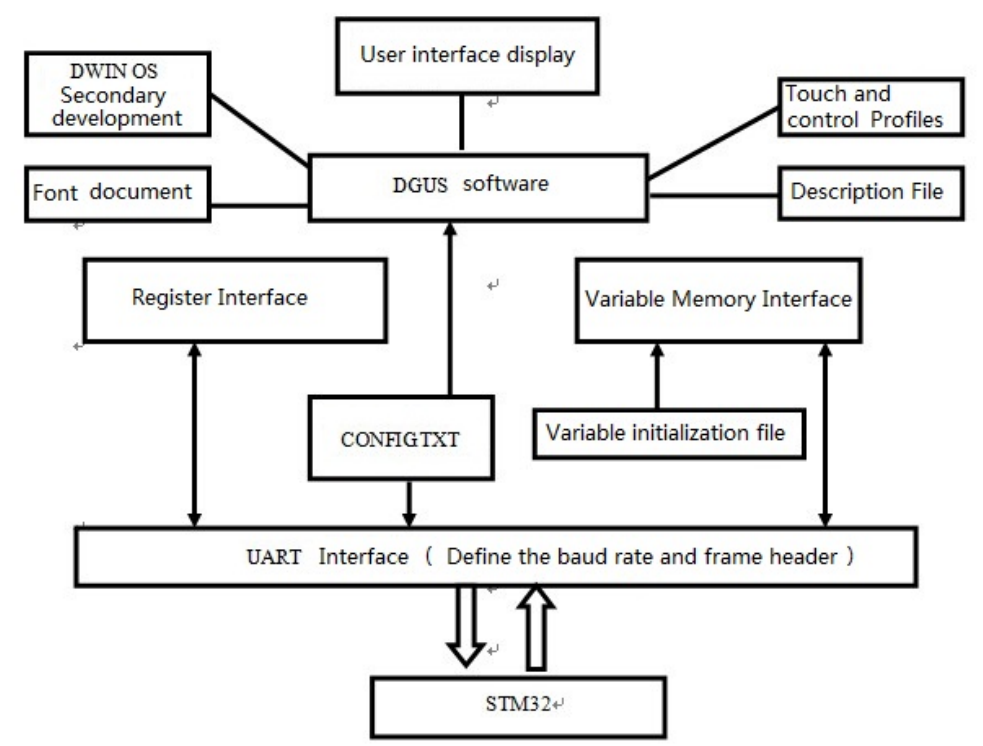

Figure 7 Functional block diagram of the power control module and man-machine interface

Touch screen input/output interface design software

The igniting power supply system has a complete detection function for the airbag, which can realize the detection of delay of time, pulse and long time power reliability. At the same time, it has the functions of the high speed camera and flash synchronous trigger and the design of the corresponding input/output interface. The delay time of the pulse current is in seconds and it can be set manually in the range of 10s. Pulse current time can be set manually in the range 5 to $10 \mathrm{~ms}$; Figure 8 is the single-channel interface in the test. Figure 9 is a multi-channel and reliability test interface design.

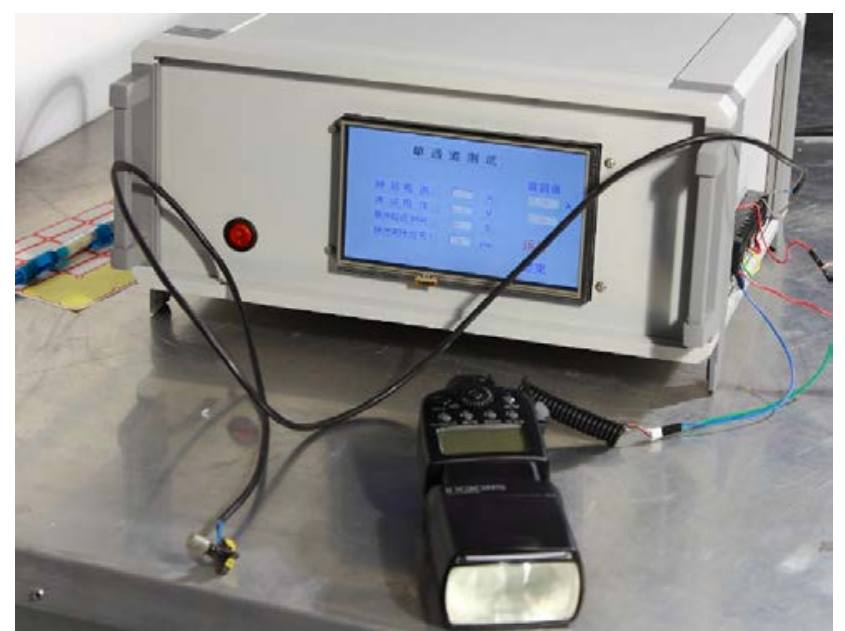

Figure 8 Working diagram of site single-channel ignition

\section{Conclusions}

After the test of the actual airbag, the design of the static igniting power supply of the airbag completely meet the design requirements. Compared with similar projects in China and abroad, the project has the advantages of low cost, rich testing functions, flexible software design, etc., ${ }^{[2]}$ The numerical control power supply has the following features:

(1) To realize the airbag deployment time, adjustable current parameters, igniting constant flow can be set and can be accurate to $0.1 \mathrm{~A}$;

(2) The time delay of pulse current is in seconds, it can be set manually in the range of 10 seconds and it can be set manually if the duration of the pulse current within 5 to $10 \mathrm{~ms}$; 

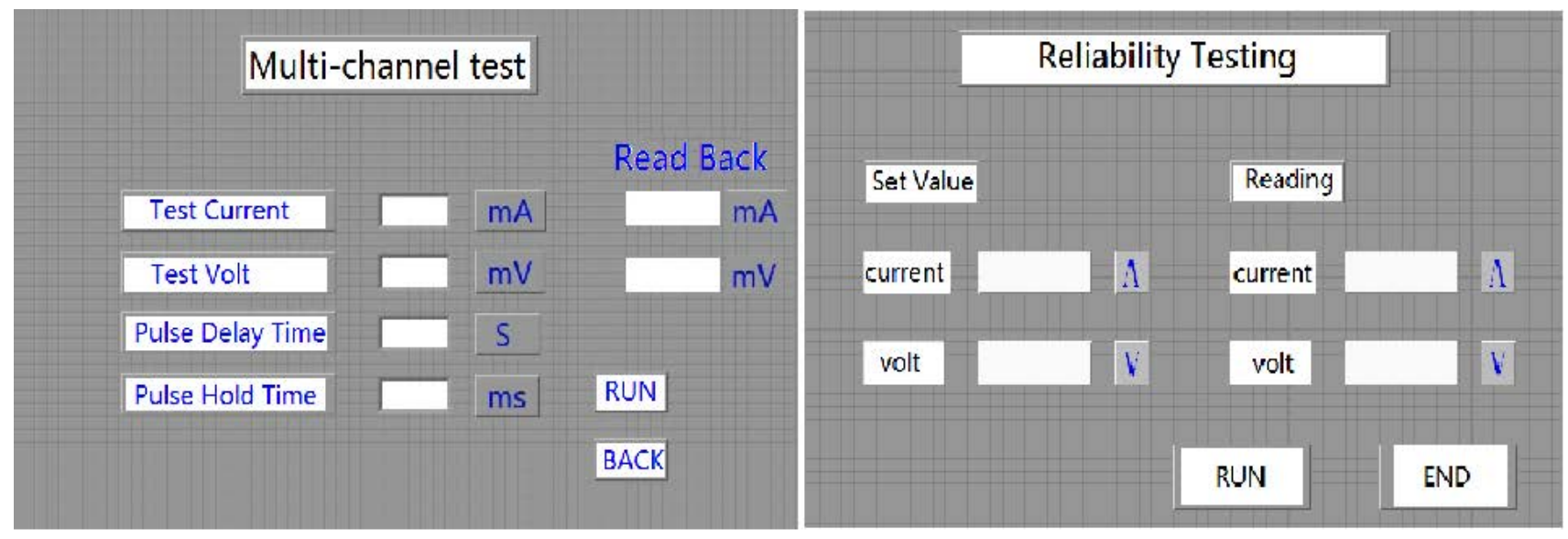

Figure 9 Multi-channel test input and reliability test interface

(3) To realize man-machine interaction function of controlling parameters, all the parameters can be input through the touch screen, with output parameters and real-time monitoring display;

(4) With complete safety protection measures, the system has power down protection function and intelligent temperature control protection function;

(5) The system has a complete detection function for the airbag, which can realize the detection of the delay of time, pulse and long time power reliability, as well as high speed camera and flash synchronous trigger function at the same time.

Due to the limited project design time, it needs to further study the airbag test parameters and evaluate the method, so that the airbag-igniting power supply can be more in line with the actual production and related standards.

\section{References}

[1] Chen Shouwen, Cheng Yi. Research and Development of Automobile Airbag [J] Automobile Research and Development, 2002, (2).

[2] Wang Xuefang, Yang Junbo, Zhang Honghai. The Automotive Airbags Intelligent Control System Design [J]. Machinery and Electronics, 2005, (11).

[3] Chen Jianjun. The Use of Digital Circuit Technology to Design a Digital Control Power [J]. Information and Computers, 2010, (6).

[4] Feng Yuhan. The NC DC Voltage Source Based on Single-chip Design [J]. Modern Electronic Technology, 2011, (9).

[5] Yang Weiming. The Numerical Control Voltage Current Regulator based on STM32 Micro Controller Design [J]. Journal of Tianjin University of Science and Technology, 2012, (10).

[6] Jing Xiaojun, Li Jianyong. The Precision of the Numerical Control Direct Current Power Supply Design [J]. The Electronic World, 2011, (10). 